\title{
MATÉRIA ORGÂNICA EM QUATRO TIPOS DE SOLOS BRASILEIROS: COMPOSIÇÃO QUÍMICA E SORÇÃO DE ATRAZINA
}

\author{
Deborah Pinheiro Dick* \\ Departamento de Físico-Química, Instituto de Química, Universidade Federal do Rio Grande do Sul. Av. Bento Gonçalves, 9500 , \\ 91501-970 Porto Alegre - RS, Brasil \\ Rosane Martinazzo \\ Departamento de Solos, Faculdade de Agronomia, Universidade Federal do Rio Grande do Sul. Av. Bento Gonçalves, 7712 , \\ 91501-970 Porto Alegre - RS, Brasil \\ Heike Knicker \\ Lehrstuhl für Bodenkunde, Technische Universität München, 85350, Freising, Germany \\ Paulo Sergio Gois Almeida \\ Centro de Ensino Superior de Rondonópolis, Av. Ari Coelho, 829, 78705-050 Rondonópolis - MT, Brasil
}

Recebido em 19/6/08; aceito em 29/6/09; publicado na web em 27/11/09

\begin{abstract}
ORGANIC MATTER IN FOUR BRAZILIAN SOIL TYPES: CHEMICAL COMPOSITION AND ATRAZINE SORPTION. Soil organic matter is the main sorptive soil compartment for atrazine in soils, followed in a minor scale by the inorganic fraction. In this study, the soil organic matter quality and atrazine sorption were investigated in four different soil types. The pedogenic environment affected the humification and therefore the chemical composition of the organic matter. The organic matter contribution to atrazine sorption was larger (60-83\%) than that of the inorganic fraction. The organic matter capacity in retaining the herbicide was favoured by a higher decomposition degree and a smaller carboxylic substitution of the aliphatic chains.
\end{abstract}

Keywords: spectroscopy; isotherms; inorganic fraction.

\section{INTRODUÇÃO}

A atrazina [2-cloro-4-etilamino-6-isopropilamino-s-triazina] é um dos herbicidas mais utilizados mundialmente, embora já tenha sido proibida sua utilização em alguns países. ${ }^{1}$ Esse herbicida seletivo é indicado para o controle de plantas invasoras anuais dicotiledôneas e algumas monocotiledôneas nas culturas de milho (Zea mays), cana-de-açúcar (Saccharum officinarum) e sorgo (Sorghum bicolor), com aplicação em pré ou pós-emergência. ${ }^{2}$ Devido à sua ampla utilização e persistência no ambiente, a atrazina e seus metabólitos são frequentemente encontrados em águas superficiais e subterrâneas. ${ }^{1}$

A disponibilidade da atrazina e de seus metabólitos para os corpos hídricos, plantas e micro-organismos está diretamente relacionada à sua sorção. ${ }^{1}$ Esse fenômeno controla a atividade das moléculas de pesticidas em solução sendo determinante nos processos de degradação, ${ }^{3,4}$ persistência, ${ }^{5}$ transporte e lixiviação ${ }^{4,6,7}$ e eficiência agronômica. ${ }^{8}$

$\mathrm{O}$ principal sorvente da atrazina em solos e em água é a matéria orgânica ${ }^{6,8}$ e, em geral, solos com maior teor de matéria orgânica apresentam maior sorção de atrazina. A intensidade de sorção de pesticidas em solos é comumente avaliada pelos coeficientes de partição $\left(K_{d}\right)$ e de Freundlich $\left(\mathrm{K}_{\mathrm{f}}\right){ }^{9}{ }^{910}$ No entanto, devido à importância da matéria orgânica do solo na retenção de vários xenobióticos, o índice $\mathrm{K}_{\mathrm{OC}}$ (normalização de $\mathrm{K}_{\mathrm{d}}$ pelo teor de carbono do sorvente) é utilizado para inferir-se sobre a capacidade de sorção desse compartimento no solo. ${ }^{9,10}$ $\mathrm{O}$ conhecimento do valor de $\mathrm{K}_{\mathrm{OC}}$ para um dado agroquímico assume importância em estudos de avaliação de impacto ambiental uma vez que é empregado para o cálculo do índice ERI (Environmental risk index). ${ }^{11}$

$\mathrm{O}$ amplo intervalo de valores para o índice $\mathrm{K}_{\mathrm{OC}}$ obtido para solos brasileiros indica que não apenas a quantidade de matéria orgânica, porém também a sua composição química afeta a capacidade de sorção de atrazina do solo. ${ }^{10,12,13}$ Recentemente, em estudo realizado

\footnotetext{
*e-mail: dpdick@iq.ufrgs.br
}

com os pesticidas carbaril e fosalona, foi observado que a natureza molecular da matéria orgânica, determinada por espectroscopia de ${ }^{13} \mathrm{C}$ RMN, afetou a sorção desses compostos. ${ }^{14}$ No entanto, em relação à especificidade dos grupos funcionais da matéria orgânica pela atrazina, os resultados obtidos são escassos e têm sido divergentes. Enquanto alguns trabalhos evidenciaram que a maior sorção de atrazina ocorreu em solos cuja matéria orgânica possuía predominância de grupamentos alifáticos e menor proporção de grupamentos carboxílicos, ${ }^{15}$ outros estudos realizados com extratos húmicos mostraram a importância dos grupos aromáticos no processo. ${ }^{16}$

Em função de sua elevada área superficial específica (ASE) e da diversidade de grupos funcionais, a matéria orgânica pode interagir com as moléculas orgânicas de diferentes formas. Os possíveis mecanismos envolvidos na interação entre herbicidas de baixa solubilidade em água, como a atrazina, e a matéria orgânica são ligação de hidrogênio, forças de van der Waals, interações hidrofóbicas e de transferência de carga, podendo os mesmos atuar concomitantemente na sorção de uma mesma molécula. ${ }^{17}$

A composição química da matéria orgânica é condicionada, principalmente, pelo ambiente pedogênico, vegetação e uso do solo. ${ }^{18,19}$ Em solos sob vegetação nativa, a matéria orgânica associada a solos cauliníticos tende a apresentar maior proporção de estruturas tipo carboidratos, enquanto que a matéria orgânica de solos esmectíticos é mais rica em grupamentos aromáticos e carboxílicos. ${ }^{20,21}$

A contribuição da fração argila, embora menos expressiva que a da matéria orgânica, para a sorção da atrazina foi estudada por alguns autores $^{17,22}$ e considerada relevante apenas em solos cujos teores de matéria orgânica são baixos. Quanto ao tipo de mineral, argilominerais do tipo 2:1 apresentam maior contribuição para a sorção de atrazina comparativamente aos minerais $1: 1{ }^{23}$ No entanto, estudos sobre sorção de atrazina em sorventes minerais têm sido realizados em minerais puros sintéticos ou isolados do solo, o que representa um sistema diferente do ambiente pedogênico. 
Tabela 1. Tipo de solo, local de coleta, condições climáticas locais, distribuição granulométrica, teor de óxidos de ferro $\left(\mathrm{Fe}_{\mathrm{d}}\right)$ e pH das amostras da camada 0 a $10 \mathrm{~cm}$ de argissolo, vertissolo, latossolo e planossolo

\begin{tabular}{|c|c|c|c|c|}
\hline Local de coleta & $\begin{array}{l}\text { Argissolo Vermelho } \\
\text { Eldorado do Sul, } \\
30^{\circ} \mathrm{S}, 51^{\circ} \mathrm{W}\end{array}$ & $\begin{array}{c}\text { Vertissolo Ebânico } \\
\text { Aceguá, } \\
31^{\circ} \mathrm{S}, 54^{\circ} \mathrm{W}\end{array}$ & $\begin{array}{c}\text { Latossolo Vermelho } \\
\text { Ijuí, } \\
28^{\circ} \mathrm{S}, 53^{\circ} \mathrm{W}\end{array}$ & $\begin{array}{c}\text { Planossolo Háplico } \\
\text { Pelotas, } \\
31^{\circ} \mathrm{S}, 52^{\circ} \mathrm{W}\end{array}$ \\
\hline $\operatorname{TMA}^{\mathrm{I}}\left({ }^{\circ} \mathrm{C}\right)$ & 18 & 17 & 18 & 18 \\
\hline $\mathrm{PMA}^{\mathrm{II}}\left(\mathrm{mm} \mathrm{ano}^{-1}\right)$ & 1310 & 1264 & 1731 & 1406 \\
\hline Areia $\left(\mathrm{g} \mathrm{kg}^{-1}\right)$ & 496 & 51 & 134 & 590 \\
\hline Silte $\left(\mathrm{g} \mathrm{kg}^{-1}\right)$ & 291 & 569 & 270 & 325 \\
\hline Argila $\left(\mathrm{g} \mathrm{kg}^{-1}\right)$ & 213 & 380 & 596 & 85 \\
\hline Mineralogia da fração argila ${ }^{\mathrm{III}}$ & $\mathrm{Ct}, \mathrm{Hm}, \mathrm{Gt}$ & Sm, Mi, Qz & $\mathrm{Ct}, \mathrm{Hm}, \mathrm{Gt}$ & $\mathrm{Ct}, \mathrm{Qz}$ \\
\hline $\mathrm{Fe}_{\mathrm{d}}^{\mathrm{IV}}\left(\mathrm{g} \mathrm{kg}^{-1}\right)$ & 8,9 & 6,1 & 19,8 & 3,2 \\
\hline $\mathrm{pH}_{\mathrm{H} 2 \mathrm{O}} \mathrm{v}$ & 4,9 & 5,1 & 4,6 & 4,7 \\
\hline
\end{tabular}

ITemperatura média anual; ${ }^{30}$ IIPrecipitação média anual; ${ }^{30}{ }^{\mathrm{II}} \mathrm{Ct}$ : caulinita, Hm: hematita, Gt: goethita, Sm: esmectita, Mi: mica, Qz: quartzo; ${ }^{30}$ IVTeor de Fe extraído por $\mathrm{DCB} ;{ }^{30} \mathrm{v} \mathrm{pH}$ do solo determinado em água destilada. ${ }^{30}$

Os objetivos principais deste estudo foram estudar a composição química da matéria orgânica em diferentes ambientes pedogênicos e, investigar a contribuição da matéria orgânica do solo, e de sua qualidade, e a contribuição da fração inorgânica na sorção de atrazina, em amostras de quatro classes de solos.

\section{PARTE EXPERIMENTAL}

\section{Locais de coleta e amostragem}

As amostras foram coletadas da camada $0-10 \mathrm{~cm}$ em perfis modais de quatro solos sob vegetação nativa, representando classes de solos representativas do Estado do Rio Grande do Sul. As características dos solos e locais de coleta são apresentadas na Tabela 1. As amostras foram secas ao ar, destorroadas, passadas em peneira de $2 \mathrm{~mm}$ (TFSA) e trituradas em gral de ágata.

\section{Oxidação da matéria orgânica e remoção dos óxidos de ferro}

A remoção da matéria orgânica das amostras foi obtida tratandose $60 \mathrm{~g}$ de solo com $120 \mathrm{~mL}$ de solução $\mathrm{H}_{2} \mathrm{O}_{2} / \mathrm{H}_{2} \mathrm{O}(15: 85 \mathrm{v} / \mathrm{v})$ sob agitação magnética e temperatura constante de $60{ }^{\circ} \mathrm{C}$, até cessar o borbulhamento. Esse processo foi mantido durante 10 dias, sendo a solução oxidante reposta à medida que ocorria evaporação. Posteriormente, a amostra foi lavada cinco vezes com água destilada, seca em estufa a vácuo a $60^{\circ} \mathrm{C}$ e triturada em gral de ágata.

A extração de óxidos de $\mathrm{Fe}$ pedogênicos $\left(\mathrm{Fe}_{\mathrm{d}}\right)$ foi realizada pelo método ditionito-citrato-bicarbonato (DCB) em temperatura ambiente $\left(20\right.$ a $25^{\circ} \mathrm{C}$ ), tratando-se $60 \mathrm{~g}$ de amostra com $1200 \mathrm{~mL}$ de solução de citrato $0,3 \mathrm{~mol} \mathrm{~L}^{-1}$ e bicarbonato de sódio $1 \mathrm{~mol} \mathrm{~L}^{-1}(8: 1 \mathrm{v} / \mathrm{v})$ e $6 \mathrm{~g}$ de ditionito em frasco com tampa sob agitação durante 16 h. ${ }^{24}$ Após secagem do resíduo, procedeu-se à oxidação da matéria orgânica e do citrato residual com peróxido de hidrogênio, conforme descrito anteriormente.

\section{Caracterização das amostras}

Os teores de carbono e de nitrogênio das amostras de solo não tratadas, tratadas com peróxido e com DCB e das amostras de matéria orgânica foram determinados em duplicata por combustão seca (analisador Perkin Elmer 2400).

A ASE dos sorventes foi determinada pelo método de adsorção de água a $20 \%$ de umidade relativa, com três repetições. Aproxima- damente $1,5 \mathrm{~g}$ do sorvente foram colocados em frascos pesa-filtro e desidratados em dessecador na presença de $\mathrm{P}_{2} \mathrm{O}_{5}$ durante 10 dias. Em seguida, as amostras secas foram pesadas e colocadas novamente em dessecador com solução de acetato de K durante 10 dias e pesadas novamente. Esse método pressupõe que, nessas condições, ocorre a adsorção de uma monocamada de água e, portanto, $1 \mathrm{mg}$ de água adsorvida equivale a uma área superficial de $3,6 \mathrm{~m}^{2} .{ }^{25}$

\section{Concentração da matéria orgânica com tratamento com solução de HF $10 \%$ (v/v)}

Para concentração da matéria orgânica e remoção de íons paramagnéticos, interferentes na análise de espectroscopia de RMN, 10 $\mathrm{g}$ de solo foram tratados com $30 \mathrm{~mL}$ de HF 10\% (v/v) sob agitação mecânica por $2 \mathrm{~h}$, em duplicata. ${ }^{21}$ Após centrifugação (1529 g, 10 min) e separação do sobrenadante, o procedimento foi repetido sete vezes até o resíduo adquirir coloração escura. A matéria orgânica concentrada foi lavada cinco vezes com água deionizada, estando o $\mathrm{pH}$ do sobrenadante na última lavagem em torno de 4,0 . As amostras de matéria orgânica foram secas em estufa a $60{ }^{\circ} \mathrm{C}$ durante $24 \mathrm{~h}$ e posteriormente pesadas. A massa recuperada após tratamento com $\mathrm{HF}\left(\mathrm{M}_{\mathrm{R}}, \%\right)$ foi calculada relacionando-se a massa final (mf) com a massa inicial $(\mathrm{mi}): \mathrm{M}_{\mathrm{R}}(\%)=[\mathrm{mf} / \mathrm{mi}]$ x 100 . A relação $\mathrm{C} / \mathrm{N}$ foi calculada antes e após o tratamento com $\mathrm{HF}\left[(\mathrm{C} / \mathrm{N})_{\mathrm{HF}}\right]$ e o fator $\mathrm{R}_{\mathrm{CN}}$ foi obtido: $\mathrm{R}_{\mathrm{CN}}=(\mathrm{C} / \mathrm{N})_{\text {solo }} /(\mathrm{C} / \mathrm{N})_{\mathrm{HF}^{*}}{ }^{21}$

\section{Espectroscopia de ${ }^{13} \mathrm{C}$ RMN CP/MAS}

Espectros de ressonância magnética nuclear de ${ }^{13} \mathrm{C}$ no estado sólido $\left({ }^{13} \mathrm{C}\right.$ RMN CPMAS) das amostras de matéria orgânica foram adquiridos em espectrômetro Bruker DSX 200 (50,3 MHz) usando rotor de zircônio de $7 \mathrm{~mm}$ OD com tampas Kel-F e velocidade de rotação no MAS de $6,8 \mathrm{kHz}$. As medidas foram realizadas com tempo de contato de 1 $\mathrm{ms}$, largura do pulso de ${ }^{1} \mathrm{H}$ de $90^{\circ}$ de $6,6 \mu$ s e intervalo entre pulsos de 200 a 300 ms. Dependendo da razão sinal/ruído, 12.000 a 15.100 scans foram acumulados, sendo empregada uma função de alargamento de linha (line broadening) de 50 a $75 \mathrm{~Hz}$ no processamento dos espectros. Os deslocamentos químicos foram referenciados ao tetrametil-silano ( 0 ppm), o qual foi ajustado com glicina (C-carboxila $=176,04 \mathrm{ppm})$ e suas respectivas atribuições foram feitas segundo Knicker e Lüdeman: ${ }^{26} 0-45$ ppm, C-alquil; 45-110 ppm, C-O-alquil; 110-160 ppm, C-aromático; 160220 ppm, C-carboxílico. A proporção relativa (\%) do sinal de cada grupo funcional foi obtida por integração da respectiva região espectral com o 
software do aparelho. Bandas laterais ocorreram apenas na região entre 220-276 pm e 0 a -50 ppm e suas intensidades foram adicionadas à região de $\mathrm{C}$-aromático, para aquelas amostras nas quais foram detectadas. ${ }^{27} \mathrm{~A}$ partir dos resultados foram calculados os seguintes índices, relacionandose as respectivas áreas: C-alquil/C-O-alquil e C-alquil/C-carboxílico. ${ }^{21}$

\section{Isotermas de sorção}

O herbicida atrazina foi empregado na forma de princípio ativo ( $97 \%$ de pureza) fornecido pela Syngenta. A atrazina é um composto polar, fracamente básico, com $\mathrm{pK}_{\mathrm{a}}$ de 1,7, solubilidade em água de $33 \mathrm{mg} \mathrm{L}^{-1}$ e densidade de $1,187 \mathrm{~g} \mathrm{~mL}^{-1}$. A solução matriz de sorbato (30 $\mathrm{mg} \mathrm{L}^{-1}$ ) foi preparada a partir do princípio ativo de atrazina em meio de $\mathrm{CaCl}_{2}$ 0,01 mol L-1, sendo o sistema mantido sob agitação durante $24 \mathrm{~h}$ para garantir a solubilização do herbicida.

Amostras dos solos não tratadas, oxidadas e deferrificadas e oxidadas foram empregadas como sorventes.

As isotermas de sorção foram obtidas em duplicata empregandose $5 \mathrm{~g}$ do sorvente em $50 \mathrm{~mL}$ de solução de atrazina nas concentrações de $0 ; 7,5 ; 15 ; 21 ; 25$ e $30 \mathrm{mg} \mathrm{L}^{-1}$, em meio de $\mathrm{CaCl}_{2} 0,01 \mathrm{~mol} \mathrm{~L}^{-1}$, preparadas por diluição da solução matriz de $30 \mathrm{mg} \mathrm{L}^{-1}$, sendo o $\mathrm{pH}$ das suspensões ajustado a 6,0 com solução diluída de $\mathrm{HCl}$ ou $\mathrm{NaOH}$. Após 24 h de agitação em agitador horizontal (160 rpm), em ambiente protegido da luz e sob temperatura ambiente $\left(23-25^{\circ} \mathrm{C}\right)$ as soluções foram centrifugadas ( $1529 \mathrm{~g}, 15 \mathrm{~min}$ ) e os sobrenadantes filtrados em filtro de papel qualitativo para separação de partículas suspensas. Em seguida, procedeu-se à extração em fase sólida (SPE) da atrazina em cartuchos Envi-Chrom-P (Supelco, Belefonte, PA, USA), utilizandose $40 \mathrm{~mL}$ da amostra e, como eluente acetona grau HPLC (Carlo Erba, Fr) ajustando-se a $10 \mathrm{~mL}$ o volume final.

A concentração de atrazina foi determinada por cromatografia a gás em um cromatógrafo Shimadzu GC 17A, com detector de ionização de chama (FID) e coluna capilar SPB-5 [poly (5\% diphenyl - 95\% dimethylsiloxane)], $30 \mathrm{~m} \mathrm{x} \mathrm{0,25} \mathrm{mm} \mathrm{x} \mathrm{0,25} \mathrm{\mu m} \mathrm{(Supelco,}$ Bellefonte, USA). A análise utilizou rampa de temperatura, iniciando em $70{ }^{\circ} \mathrm{C}$ durante 5 min e, a seguir, uma rampa de aquecimento na seguinte ordem: $15^{\circ} \mathrm{C} / \mathrm{min}$ até $170{ }^{\circ} \mathrm{C}, 5^{\circ} \mathrm{C} / \mathrm{min}$ até $200{ }^{\circ} \mathrm{C}, 15^{\circ} \mathrm{C} /$ min até $280^{\circ} \mathrm{C}$ e, por fim, 3 min em temperatura constante de 280 ${ }^{\circ} \mathrm{C}$. A temperatura do injetor foi de $250{ }^{\circ} \mathrm{C}$ e do detector $300{ }^{\circ} \mathrm{C}$. O gás de arraste utilizado foi nitrogênio 5.0 (White Martins) e o volume de injeção foi de $1 \mu \mathrm{L}$ de amostra.

Para cada amostra foram realizadas três leituras cromatográficas e a média aritmética dos valores foi calculada. A diferença entre a concentração inicial (Ci) e a de equilíbrio (Ce) foi assumida como a quantidade sorvida de atrazina, calculada pela Equação 1:

$\mathrm{Q}_{\mathrm{s}}=\frac{\left(\mathrm{C}_{\mathrm{i}}-\mathrm{C}_{\mathrm{e}}\right) \times \mathrm{V}}{m}$

onde: Qs = quantidade de atrazina sorvida $\left(\mathrm{mg} \mathrm{kg}^{-1}\right), \mathrm{v}=$ volume de solução (L) e $\mathrm{m}=$ massa do sorvente $(\mathrm{kg})$.

A curva padrão de atrazina foi obtida com cinco soluções de concentração conhecida em $\mathrm{CaCl}_{2} 0,01 \mathrm{~mol} \mathrm{~L}^{-1}$ no intervalo de 0-30 $\mathrm{mg} \mathrm{L}^{-1}$. Obteve-se uma relação linear entre a massa de atrazina e a integração da área do pico cromatográfico $\left(r^{2}=0,98\right)$.

\section{Tratamento dos dados}

Aos dados de sorção foi aplicada a Equação de Freundlich, Qs $=\mathrm{K}_{\mathrm{f}} * \mathrm{Ce}^{\text {nf }}$, onde $\mathrm{K}_{\mathrm{f}}$ e $\mathrm{n}_{\mathrm{f}}$ são constantes empíricas.

Para o cálculo do coeficiente de distribuição $\mathrm{K}_{\mathrm{d}}$, também denominado coeficiente de partição, foi empregada a Equação 2, considerando-se a concentração de equilíbrio de $7 \mathrm{mg}$ atrazina $\mathrm{L}^{-1}$, valor próximo da menor concentração de atrazina determinada experimentalmente para os diferentes sistemas desse estudo. O valor de Qs para esta concentração foi calculado a partir da equação da reta obtida para cada sorvente $(r>0,95$; $p \leq 0,01)$, abrangendo os pontos experimentais $(n=5)$ em que atrazina foi adicionada ao sistema. Desta forma, buscou-se obter o índice Kd em condições mais próximas possíveis da dose recomendada, e manter a uniformidade do cálculo nos diferentes sistemas sorbato-sorventes.

$\mathrm{K}_{\mathrm{d}}=\frac{\mathrm{Qs}}{\mathrm{Ce}}$

onde: $\mathrm{K}_{\mathrm{d}}=$ coeficiente de distribuição $\left(\mathrm{L} \mathrm{kg}^{-1}\right)$, Qs = quantidade de atrazina sorvida $\left(\mathrm{mg} \mathrm{kg}^{-1}\right)$ e $\mathrm{Ce}=$ concentração de atrazina na solução de equilíbrio $\left(\mathrm{mg} \mathrm{L}^{-1}\right)$.

Com base nos valores de $\mathrm{K}_{\mathrm{d}}$, determinou-se o índice $\mathrm{K}_{\mathrm{OC}}$ (Equação 3), que normaliza o $K_{d}$ para o teor de carbono total do sorvente:

$\mathrm{K}_{\mathrm{oc}}=\frac{\mathrm{K}_{\mathrm{d}}}{\mathrm{C}} * 1000$

onde: $\mathrm{K}_{\mathrm{OC}}=$ coeficiente de partição na fração orgânica $\left(\mathrm{L} \mathrm{kg}^{-1}\right)$ e $\mathrm{C}=$ teor de carbono do sorvente $\left(\mathrm{g} \mathrm{kg}^{-1}\right)$.

A contribuição da matéria orgânica $\left(\mathrm{C}_{\mathrm{MOS}}\right)$ para a sorção de atrazina no solo foi estimada considerando-se o valor de $\mathrm{K}_{\mathrm{d}}$ obtido no sorvente não tratado e no sorvente oxidado com $\mathrm{H}_{2} \mathrm{O}_{2}$ :

$\mathrm{C}_{\mathrm{MOS}}(\%)=\frac{\left.\mathrm{K}_{\mathrm{d}} \text { (não tratado }\right)-\mathrm{K}_{\mathrm{d}}\left(\mathrm{H}_{2} \mathrm{O}_{2}\right)}{\left.\mathrm{K}_{\mathrm{d}} \text { (não tratado }\right)} \times 100$

No latossolo e no planossolo, para a estimativa da contribuição da fração inorgânica deferrificada $\left(\mathrm{C}_{\mathrm{FID}}\right)$ foi realizado procedimento semelhante, relacionando-se o valor de Kd da amostra tratada com $\mathrm{DCB}+\mathrm{H}_{2} \mathrm{O}_{2}$ com o da amostra não tratada (Equação 5). Para a contribuição dos óxidos de ferro $\left(\mathrm{C}_{\mathrm{OFe}}\right)$ a estimativa foi obtida pela diferença entre $\mathrm{Kd}$ da amostra tratada com $\mathrm{H}_{2} \mathrm{O}_{2}$ e o da amostra tratada com $\mathrm{DCB}+\mathrm{H}_{2} \mathrm{O}_{2}$ (Equação 6).

$\mathrm{C}_{\mathrm{FID}}(\%)=\frac{\mathrm{K}_{\mathrm{d}}\left(\mathrm{DCB}+\mathrm{H}_{2} \mathrm{O}_{2}\right)}{\mathrm{K}_{\mathrm{d}}(\text { não tratado })} \times 100$

$\mathrm{C}_{\mathrm{OFe}}(\%)=\frac{\mathrm{K}_{\mathrm{d}}\left(\mathrm{H}_{2} \mathrm{O}_{2}\right)-\mathrm{K}_{\mathrm{d}}\left(\mathrm{DCB}+\mathrm{H}_{2} \mathrm{O}_{2}\right)}{\mathrm{K}_{\mathrm{d}}(\text { não tratado })} \times 100$

Nos sorventes argissolo e vertissolo o valor de $\mathrm{K}_{\mathrm{d}}\left(\mathrm{DCB}+\mathrm{H}_{2} \mathrm{O}_{2}\right)$ superou o de $\mathrm{K}_{\mathrm{d}}\left(\mathrm{H}_{2} \mathrm{O}_{2}\right)$ e a contribuição da fração inorgânica deferrificada foi estimada pela diferença entre $100 \%$ e $\mathrm{C}_{\mathrm{MOs}}$. A eventual contribuição dos óxidos de ferro não foi considerada.

\section{RESULTADOS E DISCUSSÃO}

Características dos sorventes e composição química da matéria orgânica

O teor de $\mathrm{C}$ dos solos estudados variou entre $12 \mathrm{e} 33 \mathrm{~g} \mathrm{~kg}^{-1}$, e a relação $\mathrm{C} / \mathrm{N}$ entre 9 e 12 (Tabela 2). A oxidação com $\mathrm{H}_{2} \mathrm{O}_{2}$ removeu de 85 a $97 \%$ do carbono orgânico do solo, enquanto o tratamento com DCB+ $\mathrm{H}_{2} \mathrm{O}_{2}$ foi mais eficiente na remoção de matéria orgânica (Tabela 2). Possivelmente isso se deva ao rompimento de microagregados pelo tratamento com DCB, expondo os compostos orgânicos que estavam originalmente protegidos ao ataque com $\mathrm{H}_{2} \mathrm{O}_{2}$ nas amostras não deferrificadas. 
Tabela 2. Teor de carbono e de nitrogênio, relação $\mathrm{C} / \mathrm{N}$ e área superficial específica (ASE) das amostras não-tratadas (solo), das amostras tratadas com $\mathrm{H}_{2} \mathrm{O}_{2}(\mathrm{OX})$ e das amostras tratadas com DCB e $\mathrm{H}_{2} \mathrm{O}_{2}$ $(\mathrm{OX}+\mathrm{DCB})$; teor de $\mathrm{C}$ e de $\mathrm{N}$, relação $\mathrm{C} / \mathrm{N}$, fator $\mathrm{R}_{\mathrm{CN}}$ e recuperação de massa $\left(\mathrm{M}_{\mathrm{R}}\right)$ após tratamento com $\mathrm{HF}$ e contribuições relativas dos compartimentos do solo para a sorção de atrazina em amostras da camada 0 a $10 \mathrm{~cm}$ de argissolo, vertissolo, latossolo e planossolo

\begin{tabular}{lcccc}
\hline & $\begin{array}{c}\text { Argissolo } \\
\text { Vermelho }\end{array}$ & $\begin{array}{c}\text { Vertissolo } \\
\text { Ebânico }\end{array}$ & $\begin{array}{c}\text { Latossolo } \\
\text { Vermelho }\end{array}$ & $\begin{array}{c}\text { Planossolo } \\
\text { Háplico }\end{array}$ \\
\hline $\mathrm{C}_{\text {solo }}\left(\mathrm{g} \mathrm{kg}^{-1}\right)$ & 14,5 & 33,1 & 23,0 & 12,2 \\
$\mathrm{~N}_{\text {solo }}\left(\mathrm{g} \mathrm{kg}^{-1}\right)$ & 1,5 & 3,0 & 2,0 & 1,4 \\
$\mathrm{C} \mathrm{N}_{\text {solo }}\left(\mathrm{g} \mathrm{kg}^{-1}\right)$ & 9,8 & 11,0 & 12,0 & 9,0 \\
$\mathrm{C}_{\mathrm{OX}}\left(\mathrm{g} \mathrm{kg}^{-1}\right)$ & 1,6 & 4,9 & 2,6 & 1,4 \\
$\mathrm{C}_{\mathrm{OX}+\mathrm{DCB}}\left(\mathrm{g} \mathrm{kg}^{-1}\right)$ & 0,8 & 1,1 & 1,0 & 0,5 \\
$\mathrm{ASE}_{\text {solo }}\left(\mathrm{m}^{2} \mathrm{~g}^{-1}\right)$ & 26,0 & 104,0 & 67,0 & 27,0 \\
$\mathrm{ASE}_{\mathrm{OX}}\left(\mathrm{m}^{2} \mathrm{~g}^{-1}\right)$ & 7,3 & 66,0 & 43,0 & 6,2 \\
$\mathrm{ASE}_{\mathrm{OX}+\mathrm{DCB}}\left(\mathrm{m}^{2} \mathrm{~g}^{-1}\right)$ & 11,0 & 15,0 & 12,0 & 3,0 \\
$\mathrm{C}_{\mathrm{HF}}\left(\mathrm{g} \mathrm{kg}^{-1}\right)$ & 24,0 & 176,0 & 154,0 & 18,0 \\
$\mathrm{~N}_{\mathrm{HF}}\left(\mathrm{g} \mathrm{kg}^{-1}\right)$ & 2,0 & 14,4 & 11,3 & 1,8 \\
$\mathrm{C} \mathrm{N}_{\mathrm{HF}}$ & 12,0 & 12,0 & 14,0 & 10,0 \\
$\mathrm{R}_{\mathrm{CN}}$ & 0,82 & 0,90 & 0,86 & 0,88 \\
$\mathrm{M}_{\mathrm{R}}(\%)$ & 49,0 & 13,0 & 8,9 & 55,0 \\
\hline & Contribuição para a sorção de atrazina $(\%)$ \\
\hline Matéria orgânica & 56 & 78 & 74 & 85 \\
Fração inorgânica & 44 & 22 & 22 & 8 \\
deferrificada $_{\text {Óxidos de ferro }}$ & $<1$ & $<1$ & 4 & 8 \\
\hline
\end{tabular}

O vertissolo apresentou a maior ASE (Tabela 2), o que é devido à presença de minerais do tipo 2:1 nessa amostra, os quais possuem elevada ASE quando comparados aos argilominerais 1:1. ${ }^{28}$ Entre os solos de carga de superfície variável, onde predomina a caulinita na fração argila, a ASE foi maior no latossolo, que apresenta maior teor de argila (Tabela 1). ${ }^{29}$

No argissolo e no planossolo a ASE diminuiu 72 e $77 \%$, respectivamente, após oxidação com $\mathrm{H}_{2} \mathrm{O}_{2}$, evidenciando a importante contribuição da matéria orgânica à superfície específica (Tabela 2). $\mathrm{O}$ vertissolo e o latossolo apresentaram menor decréscimo de ASE (36\%) após esse tratamento, o que se deve à contribuição dos minerais 2:1 e ao elevado teor de argila, respectivamente. A deferrificação seguida da oxidação da matéria orgânica $\left(\mathrm{DCB}+\mathrm{H}_{2} \mathrm{O}_{2}\right)$, provocou uma redução entre 50 e $77 \%$ na ASE do vertissolo, do latossolo e do planossolo em comparação às amostras oxidadas (Tabela 2), evidenciando a contribuição dos óxidos de ferro na ASE.

Os teores de $\mathrm{C}$ e de $\mathrm{N}$ aumentaram em todas as amostras após tratamento com solução de HF 10\%, como resultado da dissolução dos silicatos e óxidos (Tabela 2). A relação C/N aumentou após esse tratamento como consequência de perdas seletivas de compostos nitrogenados. Entretanto, essas perdas não foram significativas uma vez que os valores de $R_{C N}$ não foram inferiores a $0,8 .{ }^{21}$ A recuperação de massa após tratamento com $\mathrm{HF}\left(\mathrm{M}_{\mathrm{R}}\right)$ variou amplamente nas amostras estudadas ( 9 a 49\%) (Tabela 2) e se correlacionou inversamente com o teor de fração argila $(r=-0,93 ; p=0,06)$, confirmando que o tratamento com HF solubiliza preferencialmente minerais contidos nessa fração granulométrica.

A composição química da matéria orgânica dos solos, determinada a partir dos espectros de $\mathrm{RMN}$ de ${ }^{13} \mathrm{C}$, é dominada pelos grupos $\mathrm{C} \mathrm{O}$-alquil seguido de grupos C alquil (Tabela 3, Figura 1). A matéria orgânica do planossolo apresentou a maior proporção de grupos C O-alquil (53\%) e a menor proporção de $\mathrm{C}$ carboxílico (7\%) dos solos estudados. Esses resultados estão relacionados com a elevada precipitação pluviométrica durante o inverno e a insuficiente drenagem desse solo, que conduzem a um ambiente pouco oxidante, reduzindo a taxa de humificação. ${ }^{15,30} \mathrm{O}$ argissolo e vertissolo apresentaram os menores teores de $\mathrm{C} \mathrm{O}$-aquil e os maiores de $\mathrm{C}$ alquil do grupo estudado, indicando ser a matéria orgânica desses solos as mais decompostas do grupo. ${ }^{21}$ No latossolo foi observado comportamento contrário: a proporção de grupos C-O-alquil foi elevada e a de C-alquil foi a menor do grupo. Resultados semelhantes aos da composição química da matéria orgânica do argissolo e do latossolo foram observados anteriormente em outros solos sob campo nativo. ${ }^{21}$

Tabela 3. Distribuição dos grupos funcionais de carbono determinados por espectroscopia de ${ }^{13} \mathrm{C}$ RMN CP/MAS em amostras de matéria orgânica da camada 0 a $10 \mathrm{~cm}$ de argissolo, vertissolo, latossolo e planossolo

Tipo de C -

Deslocamento químico (ppm)

Argissolo Vertissolo Latossolo Planossolo Vermelho Ebânico Vermelho Háplico

\begin{tabular}{lcccc}
\hline \multicolumn{5}{c}{$\%$} \\
C alquil, 0-45 & 31,0 & 35,0 & 24,0 & 30,0 \\
C O-alquil, 45-110 & 47,0 & 46,0 & 50,0 & 53,0 \\
C aromático, 110-160 & 11,0 & 10,0 & 16,0 & 10,0 \\
C carboxílico, 160-220 & 11,0 & 9,0 & 10,0 & 7,0 \\
C alquil/C O-alquil & 0,66 & 0,76 & 0,48 & 0,57 \\
C alquil/C carboxílico & 2,8 & 3,9 & 2,4 & 4,3 \\
\hline
\end{tabular}

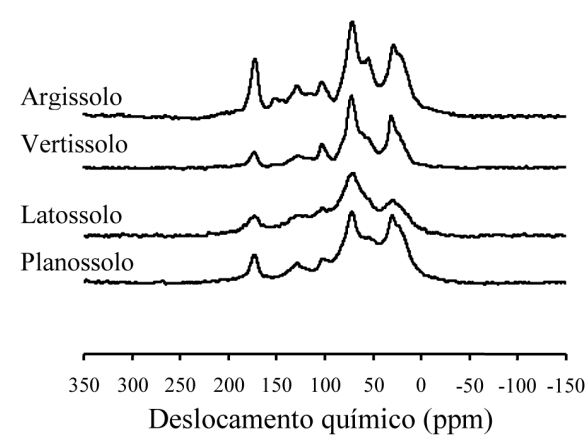

Figura 1. Espectros de ${ }^{13} \mathrm{C} R M N C P / M A S$ da matéria orgânica de argissolo, vertissolo, latossolo e planossolo

A razão $\mathrm{C}$ alquil/C O-alquil informa sobre o grau de decomposição da matéria orgânica, uma vez que os grupos $\mathrm{C}$ O-alquil, mais lábeis, são preferencialmente decompostos pelos micro-organismos, ocorrendo um enriquecimento concomitante de grupos alquil. ${ }^{21}$ No presente estudo essa razão decresceu na ordem vertissolo $>$ argissolo $>$ planossolo > latossolo (Tabela 3). Nos três solos de boa drenagem a variação do grau de decomposição pode ser explicada em função das interações organo-minerais, principalmente com óxidos de ferro. ${ }^{31}$ No latossolo, os maiores teores de óxidos de ferro e de fração argila (Tabelas 1 e 2) contribuem para uma maior estabilização da matéria orgânica por meio de interações organo-minerais em comparação aos outros ambientes pedogênicos,${ }^{30}$ ocorrendo uma fração orgânica com menor grau de decomposição. No vertissolo, a interação entre matéria orgânica e minerais 2:1 é fraca, e o efeito estabilizante da complexação de superfície é menor do que em solos de mineralogia $1: 1$, conforme já verificado anteriormente. ${ }^{20}$ Consequentemente, a matéria orgânica apresenta maior grau de decomposição do grupo. 
$\mathrm{O}$ índice $\mathrm{C}$ alquil/C carboxílico permite inferir sobre o tamanho da cadeia alquila, uma vez que a funcionalização da mesma acarreta uma diminuição de sua extensão. ${ }^{32}$ Os maiores valores observados para essa razão na matéria orgânica do planossolo e do vertissolo indicam que nestes as cadeias alquila são menos funcionalizadas do que no argissolo e no latossolo, cujos valores obtidos foram menores (Tabela 3). Valores da razão $\mathrm{C}$ alquil/C carboxílico da ordem de 2,2 a 2,6 obtidos para a matéria orgânica de latossolos brasileiros foram atribuídos à existência de cadeias alquila peptídicas de pequena extensão. ${ }^{2}$

\section{Comportamento sortivo e índices de sorção}

\section{Amostras de solo não-tratadas}

As curvas obtidas nos ensaios de sorção do latossolo, argissolo e planossolo apresentaram formato do tipo $\mathrm{C}$, enquanto que o vertissolo apresentou formato do tipo L (Tabela 4, Figura 2). $\mathrm{O}$ formato da isoterma neste solo indica que a saturação de sítios específicos já está ocorrendo no intervalo de concentração de atrazina empregado no ensaio.

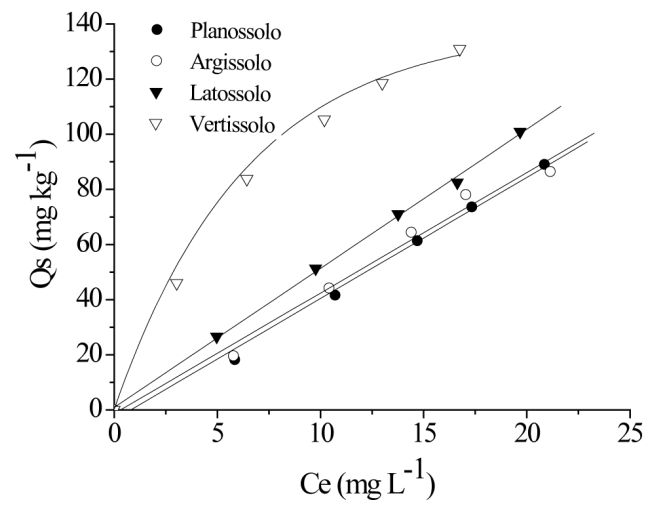

Figura 2. Isotermas de sorção de atrazina em amostras não tratadas da camada $0-10 \mathrm{~cm}$ de argissolo, vertissolo, latossolo e planossolo
A aplicação do modelo de Freundlich aos dados experimentais mostrou-se adequada $\left(r^{2} \geq 0,97\right)$, sendo que o valor de $\mathrm{K}_{\mathrm{f}}$ obtido para o vertissolo foi 5 a 10 vezes superior aos valores observados nos outros solos (Tabela 4). A maior sorção de atrazina no vertissolo foi confirmada pelo $\mathrm{K}_{\mathrm{d}}$, determinado para $\mathrm{Ce}=7 \mathrm{mg} \mathrm{L}^{-1}$, embora o $\mathrm{K}$ tenha apresentado maior sensibilidade para avaliar a sorção da atrazina, uma vez que apresentou maior amplitude de variação (Tabela 4).

Os valores de $\mathrm{K}_{\mathrm{d}}$ calculados variaram de 3,6 a $11,5 \mathrm{~L} \mathrm{~kg}^{-1}$ e diminuíram na seguinte ordem: vertissolo $>$ latossolo $>$ planossolo $\approx$ argissolo, comportamento atribuído, em parte, ao teor de carbono das amostras, cuja tendência de variação foi na mesma ordem (Tabela 2). Os valores obtidos encontram-se dentro da faixa de $\mathrm{K}_{\mathrm{d}}$ observados para sorção de atrazina em outros solos brasileiros. ${ }^{12,13}$

$\mathrm{O} \mathrm{K}_{\mathrm{d}}$ normalizado em função da ASE permite inferir sobre a densidade de sítios sortivos. ${ }^{23} \mathrm{O}$ planossolo e o argissolo apresentaram os maiores valores, seguidos do vertissolo e latossolo (Tabela 4). O maior teor de argila do vertissolo e do latossolo (Tabela 1), que é um sorvente de atrazina menos ativo do que a matéria orgânica, ${ }^{17,22}$ contribui para valores mais elevados de ASE, acarretando um menor índice $\mathrm{K}_{\mathrm{d}} / \mathrm{ASE}$. Esses resultados evidenciam que não apenas a ASE, mas também o número de sítios reativos por unidade de área interfere na sorção da atrazina,confirmando a importância da especificidade da superfície no processo.

$\mathrm{O}$ índice $\mathrm{K}_{\mathrm{OC}}$ decresceu na ordem vertissolo > planossolo > argissolo > latossolo (Tabela 4), indicando que não somente a quantidade de matéria orgânica interfere na retenção de atrazina, mas também suas características químicas e estruturais. O planossolo, comparativamente aos demais solos, apresentou o maior valor para a razão $\mathrm{C}$ alquil/ C carboxílico (Tabela 3 ).

\section{Amostras oxidadas}

Com exceção do planossolo, os valores de $\mathrm{K}_{\mathrm{f}}$ diminuíram consideravelmente após remoção da matéria orgânica (Tabela 4). $\mathrm{O}$ valor de $\mathrm{K}_{\mathrm{f}}$ do planossolo praticamente não diferiu entre os dois tipos de sorventes (não tratado e oxidado), o que se deve provavelmente ao pequeno valor desse índice observado na amostra não-oxidada. No vertissolo, adicionalmente

Tabela 4. Coeficientes de Freundlich $\left(\mathrm{K}_{\mathrm{f}}\right.$ e $\left.\mathrm{n}_{\mathrm{f}}\right)$, coeficiente de distribuição calculado para $\mathrm{Ce}=7 \mathrm{mg} \mathrm{L}^{-1}\left(\mathrm{~K}_{\mathrm{d}}\right)$ e normalização do $\mathrm{K}_{\mathrm{d}}$ em relação à área superficial específica $\left(\mathrm{K}_{\mathrm{d}} / \mathrm{ASE}\right)$ e ao carbono orgânico $\left(\mathrm{K}_{\mathrm{OC}}\right)$ de argissolo, vertissolo, latossolo e planossolo

\begin{tabular}{|c|c|c|c|c|c|c|}
\hline Solo & $\mathrm{K}_{\mathrm{f}} \mathrm{L} \mathrm{kg}^{-1}$ & $\mathrm{n}_{\mathrm{f}}$ & $\mathrm{r}^{2}$ & $\mathrm{~K}_{\mathrm{d}} \mathrm{L} \mathrm{kg}^{-1}$ & $\mathrm{~K}_{\mathrm{d}} / \mathrm{ASE} \mathrm{mL} \mathrm{\textrm {m } ^ { - 2 }}$ & $\mathrm{K}_{\mathrm{OC}} \mathrm{L} \mathrm{kg}^{-1}$ \\
\hline & \multicolumn{6}{|c|}{ amostras não-tratadas } \\
\hline Argissolo Vermelho & $4,1(1,4)$ & $1,01(0,12)$ & 0,97 & $3,56(0,41)$ & 0,14 & 200 \\
\hline Vertissolo Ebânico & $29,1(4,3)$ & $0,54(0,05)$ & 0,97 & $11,50(0,92)$ & 0,11 & 264 \\
\hline Latossolo Vermelho & $5,4(0,6)$ & $0,98(0,04)$ & 0,99 & $7,40(0,17)$ & 0,11 & 180 \\
\hline \multirow[t]{2}{*}{ Planossolo Háplico } & $2,5(0,3)$ & $1,17(0,04)$ & 0,99 & $4,30(0,78)$ & 0,27 & 238 \\
\hline & \multicolumn{6}{|c|}{ amostras tratadas com $\mathrm{H}_{2} \mathrm{O}_{2}$} \\
\hline Argissolo Vermelho & $1,2(0,3)$ & $1,12(0,09)$ & 0,98 & $1,58(0,14)$ & 0,22 & 650 \\
\hline Vertissolo Ebânico & $2,4(0,3)$ & $1,02(0,03)$ & 0,99 & $2,52(0,03)$ & 0,04 & 530 \\
\hline Latossolo Vermelho & $2,1(0,5)$ & $0,93(0,07)$ & 0,98 & $1,92(0,10)$ & 0,04 & 1120 \\
\hline \multirow[t]{2}{*}{ Planossolo Háplico } & $2,3(1,1)$ & $0,59(0,16)$ & 0,86 & $1,11(0,10)$ & 0,18 & 500 \\
\hline & \multicolumn{6}{|c|}{ amostras tratadas com $\mathrm{DCB}+\mathrm{H}_{2} \mathrm{O}_{2}$} \\
\hline Argissolo Vermelho & $2,1(0,6)$ & $0,92(0,10)$ & 0,97 & $1,82(0,10)$ & 0,17 & 2120 \\
\hline Vertissolo Ebânico & $3,2(0,6)$ & $0,92(0,06)$ & 0,99 & $2,83(0,10)$ & 0,19 & 2360 \\
\hline Latossolo Vermelho & $1,9(0,2)$ & $0,94(0,04)$ & 0,99 & $1,65(0,05)$ & 0,14 & 1600 \\
\hline Planossolo Háplico & $0,7(0,8)$ & $0,84(0,37)$ & 0,74 & $0,55(0,14)$ & 0,18 & 800 \\
\hline
\end{tabular}

Valores entre parênteses referem-se ao desvio padrão. 
ocorreu uma alteração na forma da isoterma, que passou a ser linear. A maior capacidade sortiva, avaliada pelo $\mathrm{K}_{\mathrm{d}}$, foi observada no vertissolo sugerindo que a esmectita contribui para a sorção de atrazina.

$\mathrm{O} \mathrm{K}_{\mathrm{d}}$ normalizado em relação a ASE ( $\mathrm{K}_{\mathrm{d}} / \mathrm{ASE}$ ) foi relativamente elevado no argissolo e planossolo (Tabela 4). Entretanto esses resultados não são conclusivos, pois, os valores baixos de ASE nestas amostras (Tabela 2) conduzem à superestimação dessa razão. Nas amostras oxidadas do latossolo e do vertissolo o valor de $0,04 \mathrm{~mL} \mathrm{~m}^{2}$ para $\mathrm{Kd} / \mathrm{ASE}$ se assemelha aos obtidos para minerais puros (esmectita, ilita e caulinita). ${ }^{23} \mathrm{O}$ índice $\mathrm{K}_{\mathrm{OC}}$ foi extremamente elevado e pode ser considerado um artefato resultante do baixo valor de $\mathrm{C}$ das amostras.

\section{Amostras oxidadas e deferrificadas}

Os valores de $\mathrm{K}_{\mathrm{f}}$ resultantes da sorção de atrazina em amostras de solos tratadas com $\mathrm{DCB}+\mathrm{H}_{2} \mathrm{O}_{2}$ foram relativamente baixos e pouco diferiram daqueles obtidos nas amostras oxidadas (Tabela 4). O mesmo comportamento foi observado para o índice $\mathrm{K}_{\mathrm{d}}$, indicando que a remoção dos óxidos de Fe pouco afeta a sorção de atrazina nesses substratos. Os valores de $\mathrm{K}_{\mathrm{OC}}$ nessas amostras foram, todavia, maiores do que nas amostras oxidadas e essa superestimação é resultante do baixo teor de $\mathrm{C}$ nas amostras (Tabela 2).

\section{Contribuição das frações do solo na sorção de atrazina}

Em todos os solos a matéria orgânica, embora presente em quantidades relativamente pequenas $\left(12 \leq \mathrm{C} \leq 33 \mathrm{~g} \mathrm{~kg}^{-1}\right)$, foi o principal sorvente de atrazina contribuindo com mais de $56 \%$ para a sorção (Tabela 2). Resultados semelhantes foram obtidos em estudos com Cambissolo Húmico onde as amostras naturais sorveram $49 \%$ da atrazina adicionada enquanto que amostras oxidadas (sem matéria orgânica) sorveram apenas $8 \% .{ }^{16}$ Em outro trabalho que avaliou a contribuição da fração argila na sorção da atrazina, a fração orgânica foi responsável por $68 \%$ da fração sorvida, enquanto a fração mineral foi responsável por $32 \% .{ }^{33}$

A fração inorgânica deferrificada contribuiu com 22 a 44\% para a sorção de atrazina no vertissolo, latossolo e argissolo, enquanto no planossolo a contribuição dessa fração foi de apenas $8 \%$ (Tabela 2). Esse solo difere dos demais por apresentar teor de fração argila muito baixo (Tabela 1). A contribuição dos óxidos de ferro foi relativamente pequena e não ultrapassou $8 \%$.

\section{CONCLUSÕES}

O ambiente pedogênico afetou a composição química da matéria orgânica dos solos analisados. O regime hidromórfico do planossolo dificultou o processo de humificação levando à ocorrência de uma matéria orgânica com baixa proporção de carboxilas e enriquecida relativamente em estruturas tipo carboidratos. Nos solos sob regime aeróbio, o mecanismo de estabilização da matéria orgânica via interação organo-mineral, foi mais eficiente nos solos com minerais de carga variável. O vertissolo, com mineralogia predominante de esmectitas, apresentou matéria orgânica com o maior grau de decomposição, indicado pela maior razão $\mathrm{C}$ alquil/C O-alquil. No latossolo, devido ao maior teor de argila e de óxidos de ferro, os grupos funcionais mais suscetíveis à decomposição (C O-alquil) foram protegidos pela formação de complexos organo-minerais e a razão $\mathrm{C}$ alquil/C O-alquil foi a menor do grupo de solos estudados. Em contrapartida, no argissolo, com menor teor de argila, a matéria orgânica apresentou grau de decomposição maior do que a do latossolo (maior razão $\mathrm{C}$ alquil/C O-alquil).

\section{AGRADECIMENTOS}

Ao CNPq pela concessão de bolsas de produtividade e de doutorado e ao DAAD e à CAPES pelo apoio financeiro ao projeto PROBRAL 111/00. Agradecemos também às valiosas contribuições do revisor.

\section{REFERÊNCIAS}

1. Arias-Estévez, M.; López-Periago, E.; Martínez-Carballo, E.; SimalGándara, J.; Mejuto, J. C.; García-Río, L.; Agr. Ecosyst. Environ. 2008, 123, 247.

2. Compêndio de Defensivos Agrícolas, Departamento de Defesa e Inspeção Vegetal: São Paulo, 2005.

3. Prata, F.; Lavorenti, A.; Regitano, J. B.; Tornisielo, V. L.; R. Bras. Ci. Solo 2000, 24, 217

4. Correia, F. V.; Macrae, A.; Gulherme, L. R. G.; Langenbach, T.; Chemosphere 2007, 67, 847.

5. Burauel, P.; Baßmann, F.; Environ. Pollut. 2005, 133, 11.

6. Correia, F. V.; Langenbach, T.; R.Bras. Ci. Solo 2006, 30, 183.

7. Ferri, M. V. W.; Vidal, R. A.; Fleck, N. G.; Cassol, E. A.; Gomes, P. A.; Pestic.: R. Ecotox. Meio Ambiente 2003, 13, 147.

8. Ferri, M. V. W.; Vidal, R. A.; Gomes, J.; Dick, D. P.; Souza, R. F.; Pesqui. Agropecu. Bras. 2002, 12, 1697.

9. D’Agostinho, A.; Flues, M.; Quim. Nova 2006, 29, 657.

10. Correia, F. V.; Mercante, F. M.; Fabrício, A. C.; Campos, T. M. P.; Vargas Jr., E.; Langenbach, T.; Pestici.: R. Ecotox. Meio Ambiente 2007, 17, 37.

11. Alister, C.; Kogan, M.; Crop Prot. 2006, 25, 202.

12. Oliveira Jr., R. S.; Koskinen, W. C.; Ferreira, F. A.; Weed Res. 2001, 41, 97.

13. Arantes, S. A. C. M.; Lima, J. M. de; Nóbrega, J. C. A.; Guilherme, L. R. G.; Julião, L. G. F.; Jesus, É. A. de; Pestic.: R. Ecotox. Meio Ambiente. 2006, 16, 101 .

14. Ahmad, R.; Nelson, P. N.; Kookana, R. S.; Europ. J. Soil. Sci. 2006, 57, 883.

15. Piccolo, A.; Conte, P.; Scheunert, I.; Paci, M.; J. Environ. Qual. 1998, $27,1324$.

16. Gomes, J.; Dissertação de Mestrado, Universidade Federal do Rio Grande do Sul, Brasil, 2002.

17. Sheng, G.; Johnston, C. T.; Teppen, B. J.; Boyd, S. A.; J. Agric. Food Chem. 2001, 49, 2899.

18. Dick, D. P.; Silva, L. B.; Inda Jr., A.V.; Knicker, H.; R. Bras. Ci. Solo 2008, 32, 2289

19. Rosa, C. M.; Castilhos, R. M. V.; Dick, D. P.; Paulleto, E. A.; Ci. Rural 2008, 38, 1589

20. Wattel-Koekkoek, E. J. W.; Van Genuchten, P. P. L.; Buurman, P.; Van Lagen, B.; Geoderma 2001, 99, 27.

21. Dick, D. P.; Gonçalves, C. N.; Dalmolin, R. S. D.; Knicker, H.; Klamt, E.; Kögel-Knabner, I.; Simões, M. L.; Martin-Neto, L.; Geoderma 2005, $124,319$.

22. Abate, G.; Masini, C.; J. Braz. Chem. Soc. 2005, 16, 936.

23. Herwig, U.; Klumpp, E.; Narres, H.; Schwuger, M. J.; Appl. Clay Sci. 2001, 18, 211

24. Dick, D. P.; Schwertmann, D.; Geoderma 1996, 74, 49

25. Quirk, J. P.; Soil Sci. 1955, 80, 423.

26. Knicker, H.; Lüdemann, H. D.; Org. Geochem. 1995, 23, 329.

27. Knicker, H,; González-Vila, F. J.; Polvillo, O.; González, J. A.; Almendros, G.; Soil Biol. Biochem. 2005, 37, 701

28. Sparks, D. L.; Environmental Soil Chemistry, 2nd ed., Academic: San Diego, 2002.

29. Almeida, P. S. G.; Dick, D. P.; Cesur em Revista 2004, 3, 91

30. Dalmolin, R. S. D.; Gonçalves, C. N.; Dick, D. P.; Knicker, H.; Klamt, E.; Kögel-Knabner, I.; Eur. J. Soil Sci. 2006, 57, 644.

31. Eusterhues, K.; Rumpel, C.; Kögel-Knabner, I.; Org. Geochem. 2005, 36, 1567.

32. Knicker, H.; Schmidt, M. I.; Koegel-Knabner, I.; Soil Biol. Biochem. 2000, 32, 241.

33. Laird, D.; Yen, P. Y.; Koskinen, W. C.; Steinheimer, T. R.; Dowdy, R. H.; Environ. Sci. Technol. 1994, 28, 1054. 